\title{
Thermodynamics, size, and dynamics of zwitterionic dodecylphosphocholine and anionic sodium dodecyl sulfate mixed micelles
}

\author{
Emilia Sikorska $^{1}$ - Dariusz Wyrzykowski ${ }^{1} \cdot$ Kosma Szutkowski $^{2,3} \cdot$ Katarzyna Greber $^{4}$. \\ Emilia A. Lubecka ${ }^{1} \cdot$ Igor Zhukov $^{3,5}$
}

Received: 22 April 2015/Accepted: 30 June 2015/Published online: 21 July 2015

(C) The Author(s) 2015. This article is published with open access at Springerlink.com

\begin{abstract}
The thermodynamic properties of micellization for dodecylphosphocholine (DPC), sodium dodecyl sulfate (SDS), and their mixtures were studied using isothermal titration calorimetry. NMR relaxation measurements were used to explore molecular mobility of the DPC-containing micelles, whereas the diffusion measurements were taken to determine the micelle size. The DPC/SDS mixed systems reveal a tendency to form two kinds of micelles in buffered solution at lower temperatures. An increase in temperature as well as the transfer of the DPC/SDS mixed micelles from buffered to unbuffered solution results in only a single-step micellization process. The average size of the DPC-containing micelles is only slightly dependent on the SDS fraction. Examination of the data of spin-spin relaxation $\left(T_{2}\right)$ shows that methylene protons on the polar headgroup of DPC and methylene protons (H1) on the hydrocarbon chain in the micellar systems studied reveal a heterogeneous dynamic behavior reflected in a two-
\end{abstract}

Electronic supplementary material The online version of this article (doi:10.1007/s10973-015-4918-0) contains supplementary material, which is available to authorized users.

Emilia Sikorska

emilia.sikorska@ug.edu.pl

1 Faculty of Chemistry, University of Gdańsk, Wita Stwosza 63, 80-308 Gdańsk, Poland

2 Faculty of Physics, Adam Mickiewicz University, Umultowska 85, 61-614 Poznań, Poland

3 Nanobiomedical Centre, Adam Mickiewicz University, Umultowska 85, 61-614 Poznań, Poland

4 Faculty of Pharmacy, Medical University of Gdańsk, Al. Gen. J. Hallera 107, 80-416 Gdańsk, Poland

5 Institute of Biochemistry and Biophysics, Polish Academy of Sciences, Pawińskiego 5a, 02-106 Warsaw, Poland component $T_{2}$ relaxation in the whole temperature range. The latter is the main constituent of the rigid interfacial layer core protecting the penetration of water into the hydrophobic interior.

Keywords DPC/SDS mixed micelles $\cdot$ Surfactants $\cdot$ ITC · NMR

$\begin{array}{ll}\text { Abbreviations } \\ \text { Cmc } & \text { Critical micelle concentration } \\ \text { DPC } & \text { Dodecylphosphocholine } \\ \text { DPFGDSTE } & \begin{array}{l}\text { Double pulsed gradient double stimulated } \\ \text { echo }\end{array} \\ \text { ITC } & \begin{array}{l}\text { Isothermal titration calorimetry } \\ \text { SDS }\end{array} \\ \text { Sodium dodecyl sulfate }\end{array}$

\section{Introduction}

The structure and activity of membrane-active peptides are closely related to the ability to associate with cell membrane [1-5]. Thus, the structural studies of such peptides by solution NMR spectroscopy require membrane-mimicking environment for proper folding and stability. And here, the structural complexity of biological membranes becomes quite a challenge. Among the most prominent membrane models suitable for the peptide-membrane interaction studies, the similarity to biological membranes decreases in the order: liposomes, bicelles, mixed micelles, and micelles [6]. The NMR studies of peptides in the presence of liposomes are usually limited to the solid state due to their considerable size. In solution NMR, the slow molecular tumbling resulting from the liposome size leads to a 
broadening of the NMR resonances, which makes achievement of a high-resolution 3D structure of the liposome-bound peptide difficult or even impossible $[7,8]$. In turn, micelles stand for a good compromise between suitable membrane model and the need for relatively fast tumbling system in view of their short rotational correlation time [9]. The most commonly used micellar membrane-mimicking systems are aqueous solutions of surfactants such as dodecylphosphocholine (DPC) and sodium dodecyl sulfate (SDS) [10-14]. DPC provides a zwitterionic surface on the micelles that adequately mimic biological membranes of the vertebrates, whereas the SDS micelles, with negatively charged headgroups, adequately imitate the bacterial membrane [15]. However, to mimic electrostatic properties of the vertebrate plasma, a membrane model characterized by a slight prevalence of the negative charge, such as that in the DPC/SDS mixed micelles, can be used as well [16-18]. The use of SDS to imitate membrane environment is not always rational because it has no structural analogues among the phospholipids that are components of biological membranes. However, SDS is the only commercially available detergent that is completely deuterated and negatively charged.

In the present work, we use the isothermal titration calorimetry (ITC) to study the micellization of DPC, SDS, and DPC/SDS mixed systems as a function of the temperature. The relative arrangement and interactions of zwitterionic DPC with anionic SDS were studied by NMR. The diffusion coefficient measurements were used to determine the size of the micellar aggregates.

\section{Materials and methods}

\section{Reagents}

The DPC was purchased from Avanti Polar Lipids, Inc, whereas SDS from Sigma-Aldrich.

\section{ITC measurements}

All ITC experiments were performed over the 293-323 K temperature range using an AutoITC isothermal titration calorimeter (MicroCal Inc. GE Healthcare, Northampton, USA). The data were analyzed with Origin 7 software (MicroCal, Inc.). Specifically, the experiment consisted of injecting $10.02 \mu \mathrm{L}$ (29 injections, $2 \mu \mathrm{L}$ for the first injection only) of a $20 \mathrm{mM}$ buffered surfactant solution into a 1.4491-mL cell containing a buffer solution (PBS, a $10 \mathrm{mM}$ phosphate buffer, $\mathrm{pH} 7.4$, containing $2.7 \mathrm{mM}$ potassium chloride and $137 \mathrm{mM}$ sodium chloride). Each injection lasted $20 \mathrm{~s}$. The titrant was injected at 4-min intervals to ensure that the titration peak returned to the baseline before the next injection. To ensure a homogeneous mixing in the cell, the stirrer speed was kept constant at $300 \mathrm{rpm}$. The same procedure was repeated for aqueous buffer-free solutions of surfactants studied at $298 \mathrm{~K}$. A calibration of the AutoITC calorimeter was carried out electrically by using electrically generated heat pulses. The $\mathrm{CaCl}_{2}$-EDTA titration was performed to check the apparatus, and the results ( $n$-stoichiometry, $K, \Delta H$ ) were compared with those obtained for the same samples (a test kit) at MicroCal Inc. GE Healthcare.

\section{Surface tension measurements}

The critical micelle concentration $(\mathrm{cmc})$ of the mixed micelles composed of DPC and SDS taken at mole ratios of 1:0, 9:1, and 5:1 was determined by plotting the surface tension as a function of the logarithm of total surfactant concentration and was found as the intersection of two lines that best fit through the pre- and post-cmc data. Concentrated solutions of the surfactants of known concentration were progressively diluted. All surfactant solutions were prepared in water purified by HLP5 system. The surface tension of each sample was determined at $298 \mathrm{~K}$ by the Du Noüy ring method on an Easy Dyne tensiometer (Krüss GmbH, Germany).

\section{NMR experiments}

\section{Diffusion measurements}

To extract information about the self-diffusion coefficients $\left(D_{\mathrm{tr}}\right)$ of the DPC-containing micelles, the DPFGDSTE (double pulsed field gradient double stimulated echo) experiments were conducted at 293, 303, and $313 \mathrm{~K}$ on an Agilent VNMRS $600 \mathrm{NMR}\left({ }^{1} \mathrm{H}\right.$ resonance frequency $599.93 \mathrm{MHz}$ ) spectrometer equipped with a 5-mm probehead (DOTY Scientific Ltd.) dedicated for diffusion measurements. The echo attenuation intensity $(I)$ as a function of gradient amplitude $(g)$ is expressed by Stejskal-Tanner equation [19]: $I=I_{0} \exp \left[-D g^{2} \gamma^{2} \delta^{2}(\Delta-\delta / 3)\right]$, where $I_{0}$ is the echo intensity in the absence of field gradient pulse, $\gamma=2.675 \times 10^{8} \mathrm{rad} \mathrm{T}^{-1} \mathrm{~s}^{-1}$ is the gyromagnetic ratio of the ${ }^{1} \mathrm{H}$ nucleus, $\delta$ is the duration of the field gradient pulse, and $\Delta$ is the time period between the two field gradient pulses. In our experiments, the diffusion time $\Delta$ was $50 \mathrm{~ms}$ and the diffusion gradient time $\delta$ had a maximum duration of $2 \mathrm{~ms}$. A single exponential decay of the integral intensity of the signal at $3.23 \mathrm{ppm}\left[\mathrm{N}\left(\mathrm{CH}_{3}\right)_{3}\right]$ versus diffusion gradients $g^{2} \gamma^{2} \delta^{2}(\Delta-\delta / 3)$ was used to determine the translational self-diffusion coefficients. 
${ }^{1} H$ and ${ }^{31} P$ relaxation measurements for DPC-containing micelles

The longitudinal $\left(T_{1}\right)$ and transverse $\left(T_{2}\right)$ relaxation times were measured on a Varian Unity Plus $500 \mathrm{MHz}$ spectrometer $\left({ }^{1} \mathrm{H}\right.$ resonance frequency $\left.499.83 \mathrm{MHz}\right)$ using the inversion recovery $(\pi-\tau-\pi / 2)$ and the Carr-Purcell-Meiboom-Gill (CPMG) pulse sequences, respectively. The protonated DPC and SDS were used in all the experiments. The 1:0, 9:1, and 5:1 DPC/SDS mixed systems were studied at a total surfactant concentration fixed at $150 \mathrm{mM}$. All NMR measurements were taken in a $10 \mathrm{mM}$ deuterated phosphate buffer ( $\mathrm{pD} 7.4$ ).

\section{Results and discussion}

\section{ITC results}

The thermodynamic properties of the micelles were determined using ITC in a $10 \mathrm{mM}$ phosphate buffer (PBS), $\mathrm{pH} 7.4$, containing $2.7 \mathrm{mM}$ potassium chloride and $137 \mathrm{mM}$ sodium chloride. In the ITC experiment, the first injections of the micellar surfactant solution into buffer solution result in a heat being the sum of three processes, i.e., dilution of micelles, demicellization and dilution of the resultant monomers. The release of counterions is included in the observed reaction but cannot be separated [20]. Once the total concentration of the surfactant in the reaction cell reaches $c m c$, a sharp change in the heat of injection is observed. At the end of the experiment, the enthalpy change is solely the result of micelle dilution without breaking down the micelles [21]. The difference between the enthalpy before and after micellization gives the enthalpy of demicellization process, $\Delta H_{\text {demic }}$. The heat of micelle formation has the opposite sign, i.e., $\Delta H_{\text {mic }}=-\Delta H_{\text {demic }}$. The calculation of the first derivative of the ITC curve with respect to the total surfactant concentration in the cell allows us to determine the critical micelle concentration $(\mathrm{cmc})$. The procedure of determining the enthalpy of micellization and $c m c$ is shown in Fig. 1.

The standard Gibbs energy of micellization process $\left(\Delta G_{\text {mic }}\right)$ is related to the critical micelle concentration $(\mathrm{cmc})$ by the following equation [22-24]:

$\Delta G_{\mathrm{mic}}=\mathrm{RT} \ln X_{\mathrm{cmc}}$

where $R T$ is the thermal energy and $X_{\mathrm{cmc}}$ is the critical micelle concentration in mole fraction units with respect to water. The entropy of micelle formation was determined from a standard thermodynamic relationship [23, 24]:

$\Delta G_{\mathrm{mic}}=\Delta H_{\mathrm{mic}}-T \Delta S_{\mathrm{mic}}$

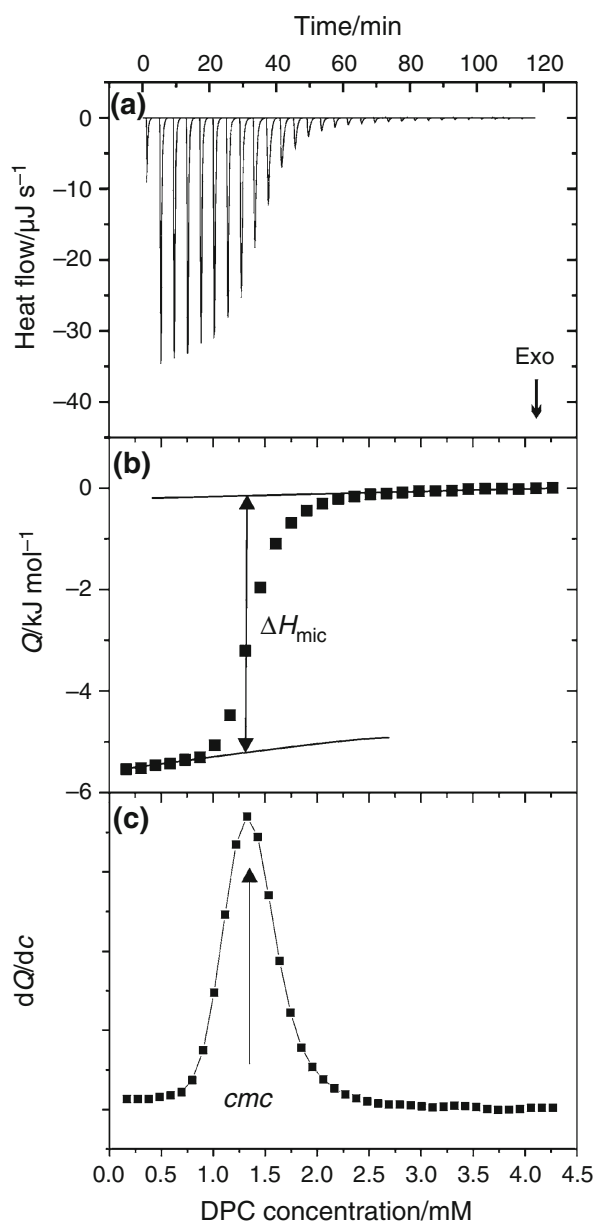

Fig. 1 ITC experiment for the determination of the $\mathrm{cmc}$ of DPC in $10 \mathrm{mM}$ PBS at pH 7.4 at $298 \mathrm{~K}$. a Raw data from ITC titration (heat flow vs. time), $\mathbf{b}$ integrated injection heats versus DPC concentration in the reaction cell, and $\mathbf{c}$ first derivative of ITC titration curve calculated

The mixed micelles in PBS reveal a two-step aggregation mechanism: cmc and $\Delta H_{\text {mic }}$

The enthalpy of micellization is the result of several processes. The endothermic contribution to the micellization is related to release of water from the "hydrophobically hydrated" nonpolar hydrocarbon chains of surfactant monomers during their transfer from solution into the micellar core. The stabilization of the monomers in the micelle, the counterion binding to the micelle, and the associated hydration give an exothermic contribution to the process [25]. Thus, the resultant enthalpy, $\Delta H_{\text {mic }}$, becomes more negative with increasing temperature. The temperature at which the enthalpy of micellization, $\Delta H_{\text {mic }}$, changes from endothermic to exothermic depends on the nature of the surfactant. 
In the case of SDS, the micellization process is always exothermic over the studied temperature range, whereas for the DPC, the micellization is endothermic $\left(\Delta H_{\text {mic }}>0\right)$ at lower temperatures and exothermic $\left(\Delta H_{\text {mic }}<0\right)$ at higher ones (Table 1). The DPC/SDS mixed micelles exhibit two inflection points in ITC curves up to a temperature of ca. $313 \mathrm{~K}$. The analysis of the first derivative of ITC curve clearly shows the presence of two extrema indicating two $c m c$ values denoted by $c m c 1$ and $c m c 2$ (Fig. 2).

The second transition region at $c m c 2$ is much broader with no abrupt change in enthalpy. Consequently, $c m c 2$ and enthalpy of the second step of micellization could be measured less accurately. The presence of two $c m c$ s indicates a two-step aggregation mechanism. Surfactants with at least two $c m c$ s are known as "multiple-cmc systems"
[26] and have previously been reported elsewhere [20, 25, 27-29]. Both steps of the micellization processes in the DPC/SDS mixed micelles are endothermic $\left(\Delta H_{\text {mic }}=-\Delta H_{\text {demic }}\right)$ at low temperatures, and due to proximity of both $c m c$ values, the separation of two reaction enthalpies is difficult (Fig. 2). In turn, over the ranges of 303-313 and 298-308 K for the 9:1 DPC/SDS and 5:1 DPC/SDS, respectively, the first step of micellization becomes gradually more exothermic, whereas the second one gradually less endothermic. This facilitates determination of the enthalpy of each step separately (Fig. 2). At higher temperatures, micellization takes place as a singlestep exothermic process with only one $c m c$ (Table 1). The similar tendency was previously observed for alkyl triphenylphosphonium bromides [29].

Table 1 Thermodynamic parameters of micelle formation for DPC, SDS, and DPC/SDS mixed micelles

\begin{tabular}{|c|c|c|c|c|c|}
\hline Micelle & $T / \mathrm{K}$ & $\mathrm{cmc} / \mathrm{mM}$ & $\Delta H_{\mathrm{mic}} / \mathrm{kJ} \mathrm{mol}^{-1}$ & $\Delta G_{\text {mic }} / \mathrm{kJ} \mathrm{mol}^{-1}$ & $T \Delta S_{\mathrm{mic}} / \mathrm{kJ} \mathrm{mol}^{-1}$ \\
\hline \multirow[t]{7}{*}{ DPC } & 293 & 1.46 & 7.80 & -25.68 & 33.48 \\
\hline & 298 & 1.36 & 4.97 & -26.29 & 31.26 \\
\hline & 303 & 1.34 & 3.22 & -26.77 & 29.99 \\
\hline & 308 & 1.33 & 0.40 & -27.23 & 27.63 \\
\hline & 313 & 1.39 & -1.99 & -27.56 & 25.57 \\
\hline & 318 & 1.42 & -4.37 & -27.94 & 23.57 \\
\hline & 323 & 1.46 & -6.59 & -28.31 & 21.71 \\
\hline \multirow[t]{7}{*}{ 9:1 DPC/SDS } & 293 & $1.05(1.70)$ & $3.40^{\mathrm{a}}$ & $-26.48(-25.31)$ & $29.88(28.71)$ \\
\hline & 298 & $0.94(1.60)$ & $2.58^{\mathrm{a}}$ & $-27.21(-25.89)$ & $29.78(28.46)$ \\
\hline & 303 & $0.89(1.46)$ & $-0.88(1.04)$ & $-27.80(-26.55)$ & $26.92(27.59)$ \\
\hline & 308 & $0.77(1.27)$ & $-2.71(0.83)$ & $-28.63(-27.35)$ & $25.92(28.17)$ \\
\hline & 313 & $0.82(1.39)$ & $-4.42(0.26)$ & $-28.93(-27.56)$ & $24.51(27.82)$ \\
\hline & 318 & 0.89 & -5.94 & -29.18 & 23.23 \\
\hline & 323 & 0.94 & -7.93 & -29.48 & 21.55 \\
\hline \multirow[t]{7}{*}{ 5:1 DPC/SDS } & 293 & $0.68(1.19)$ & $3.29^{\mathrm{a}}$ & $-27.54(-26.17)$ & $30.83(29.47)$ \\
\hline & 298 & $0.65(1.18)$ & $-0.19(1.79)$ & $-28.12(-26.64)$ & $27.93(28.44)$ \\
\hline & 303 & $0.66(1.18)$ & $-1.35(1.64)$ & $-28.55(-27.09)$ & $27.20(28.73)$ \\
\hline & 308 & $0.70(1.18)$ & $-3.22(0.88)$ & $-28.87(-27.54)$ & $25.65(28.42)$ \\
\hline & 313 & 0.74 & -5.65 & -29.20 & 23.54 \\
\hline & 318 & 0.78 & -7.63 & -29.52 & 21.89 \\
\hline & 323 & 0.85 & -9.45 & -29.76 & 20.31 \\
\hline \multirow[t]{7}{*}{ SDS } & 293 & 0.95 & -0.56 & -26.72 & 26.17 \\
\hline & 298 & 1.10 & -2.53 & -26.82 & 24.29 \\
\hline & 303 & 1.00 & -5.86 & -27.51 & 21.65 \\
\hline & 308 & 1.00 & -8.37 & -27.96 & 19.59 \\
\hline & 313 & 1.27 & -9.50 & -27.79 & 18.29 \\
\hline & 318 & 1.34 & -11.47 & -28.09 & 16.63 \\
\hline & 323 & 1.46 & -13.41 & -28.31 & 14.89 \\
\hline
\end{tabular}

The mixed micelles exhibit two inflection points in ITC curves up to a temperature of ca. $313 \mathrm{~K}$. The parameters in parentheses correspond to the second step of micellization process

${ }^{\text {a }}$ Both steps of the micellization processes in the DPC/SDS mixed micelles are endothermic, and due to proximity of both $\mathrm{cmc}$ values, the separation of two reaction enthalpies is difficult. Consequently, in these cases only average $T \Delta S_{\text {mic }}$ values were calculated from the total heat of micellization 

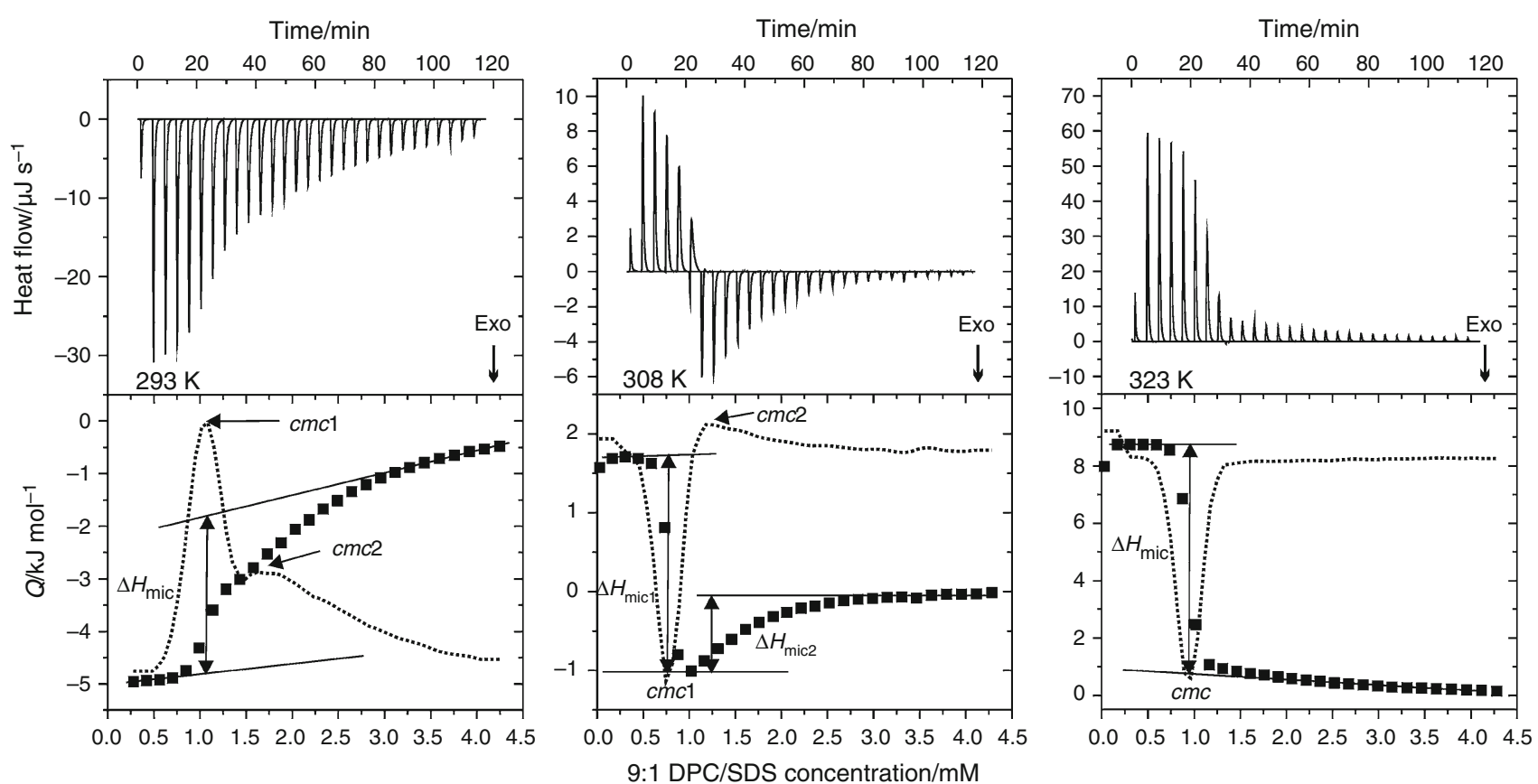

Fig. 2 ITC experiment for the determination of the $c m c$ of the 9:1 DPC/SDS in $10 \mathrm{mM}$ PBS at $\mathrm{pH} 7.4$ at three temperatures. Top panel corresponds to the experimental raw data (heat flow vs. time), bottom panel corresponds to the integrated injection heats and the first

The $c m c$ value of pure DPC in $10 \mathrm{mM}$ PBS at $298 \mathrm{~K}$ is $1.36 \mathrm{mM}$ and is consistent with the recent results [30]. In the case of SDS, $c m c$ depends strongly on the solution ionic strength. Previous studies have shown that in water, the cmc of SDS is ca. $8 \mathrm{mM}$, whereas in buffered solution or in the presence of $150 \mathrm{mM} \mathrm{NaCl}$, it decreases to about $1-2.5 \mathrm{mM}[23,30-33]$. In our studies, the $\mathrm{cmc}$ of SDS in PBS at $298 \mathrm{~K}$ is $1.1 \mathrm{mM}$, which is consistent with the reported data. Addition of SDS to the DPC micelle results in a decrease in the $c m c$ values to 0.94 and $0.65 \mathrm{mM}$ at $298 \mathrm{~K}$ for the 9:1 and 5:1 DPC/SDS systems, respectively (assuming the $c m c 1$ values for both mixtures). As it has been earlier reported, the $c m c$ for the mixture of two oppositely charged surfactants becomes significantly smaller than that of separate surfactants due to mutual association of the surfactants induced by electrostatic attraction [34-36]. The present results can also be explained assuming that the association between the zwitterionic DPC and anionic SDS is favorable due to electrostatic attractions between the cationic fragment of DPC and the dodecyl sulfate anion. Consequently, those electrostatic interactions are considered to be largely responsible for the synergism in the formation of the DPC/SDS mixed micelles.

The $c m c$ data of the micelles studied are summarized as a function of temperature in Fig. 3. In pure SDS, the distinct presence of the minimum in the $c m c$ (vs. temperature) derivative of the ITC titration curve calculated from the interpolated values. Two-step aggregation mechanism is observed for the 9:1 DPC/SDS system over the temperature range of 293-308 K. Increasing temperature results in a single-step micellization process

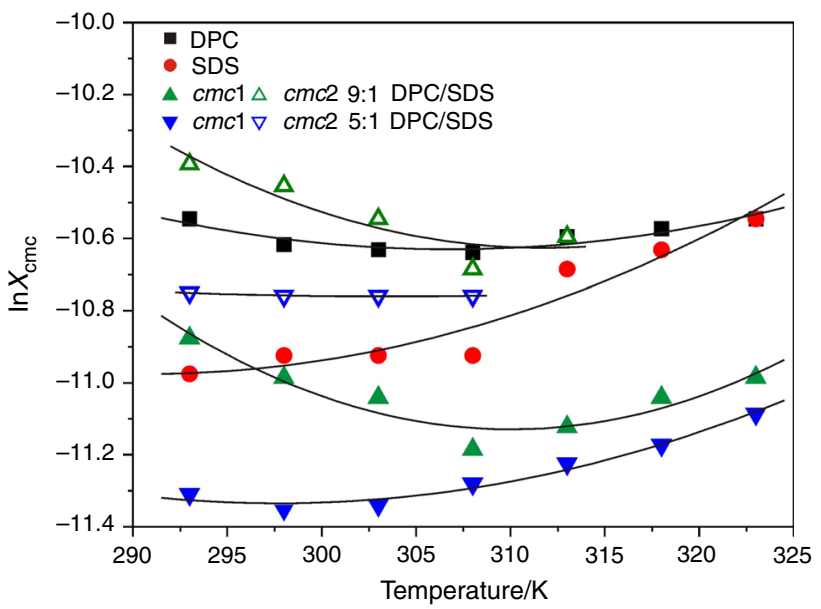

Fig. 3 Temperature-dependent values of $\ln X_{\text {cmc }}$ for the micelles studied in PBS pH 7.4 by calorimetric methods. The data were fitted with a second-degree polynomial function

is not observed up to the lower studied temperature of $293 \mathrm{~K}$. For both DPC and 9:1 DPC/SDS micelles, the minimum of $c m c$ occurs at $308 \mathrm{~K}$ and is shifted to $297 \mathrm{~K}$ for 5:1 DPC/SDS (taking into account the $c m c 1$ for the mixed micelles). In the case of the 9:1 DPC/SDS, the value of $c m c 2$ is distinctly greater than $c m c$ s of both DPC and SDS surfactants at lower temperatures. However, at 308 and $313 \mathrm{~K}$, it is very close to that obtained for pure DPC. In turn, the position of the $c m c 2$ for the 5:1 DPC/SDS is 
always between $c m c$ values determined for pure DPC and SDS and does not change with increasing temperature. It is worth emphasizing that the $c m c$ determined previously in PBS solution for 9:1 DPC/SDS system at $298 \mathrm{~K}(1.35 \mathrm{mM})$ [30] has been found to be an average of both $\mathrm{cmcs}$ obtained by calorimetry in this paper.

The temperatures $\left(T_{0}\right)$ at which the enthalpy of micellization vanishes and the process is entropically driven are derived from the intersection of second polynomial approximation of the temperature dependence of $\Delta G_{\text {mic }}$ and $-T \Delta S_{\text {mic }}$ terms $\left(\Delta G_{\text {mic } 1}\right.$ and $-T \Delta S_{\text {mic } 1}$ for the DPC/SDS mixed micelles) as shown in Fig. 4. The $T_{0}$ temperatures were found to be $309,302,300$, and $292 \mathrm{~K}$ for DPC, 9:1 DPC/SDS, 5:1 DPC/SDS, and SDS, respectively. As seen, it shifts to lower values as the concentration of negatively charged SDS increases. Application of the same method to the published ITC data for micellization of SDS in $0.1 \mathrm{M} \mathrm{NaCl}$ and $50 \mathrm{mM}$ HEPES buffer of $\mathrm{pH} 7.4$ gives $T_{0}$ values at around $292 \mathrm{~K} \mathrm{[23]} \mathrm{and} 291 \mathrm{~K}$ [32], respectively. The temperatures $T_{0}$ deduced from the enthalpy curves are more or less consistent with those obtained from the temperature dependence of $c m c$ s. However, like previous reporting [23], if the $c m c$ is a directly measured
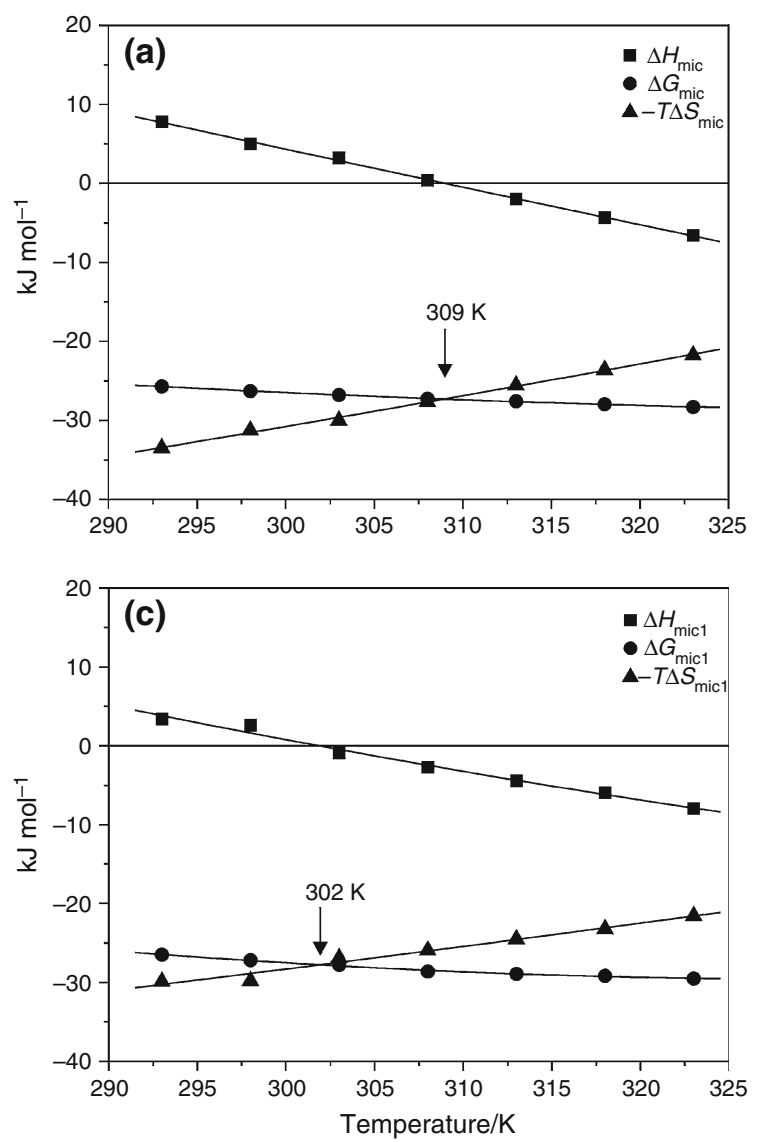

quantity, the minimum may not be easily detected and the transition temperature $\left(T_{0}\right)$ determined from the temperature dependence of $\Delta H_{\text {mic }}$ provides a more precise determination for the temperature of the $c m c$ minimum.

\section{Heat capacity of micellization}

The first derivative of $\Delta H_{\text {mic }}$ with respect to temperature at constant pressure yields the change in heat capacity $\Delta C_{\mathrm{p}, \text { mic }}$, and this, in turn, at $298 \mathrm{~K}$ is shown as a linear function of the hydrophobic surface in micelle not exposed to the water. The number of "dry" hydrogen atoms $\left(n_{\mathrm{H}}\right)$ in the micelle of the surfactant with a straight hydrocarbon chain, which are not in contact with water, is expressed by the following equation [23, 37]:

$\Delta C_{p, \text { mic }}=33 n_{\mathrm{H}}\left(\mathrm{Jmol}^{-1} \mathrm{~K}^{-1}\right)$

In the present study, all the $\Delta C_{\mathrm{p} \text {,mic }}$ values were found to be negative throughout the whole temperature range, which is normally observed for self-association of surfactants leading to micelle formation.

Figure 5 illustrates the temperature dependence of $\Delta C_{\mathrm{p}, \text { mic }}\left(\Delta C_{\mathrm{p}, \text { mic } 1}\right.$ for the DPC/SDS mixed micelles). The
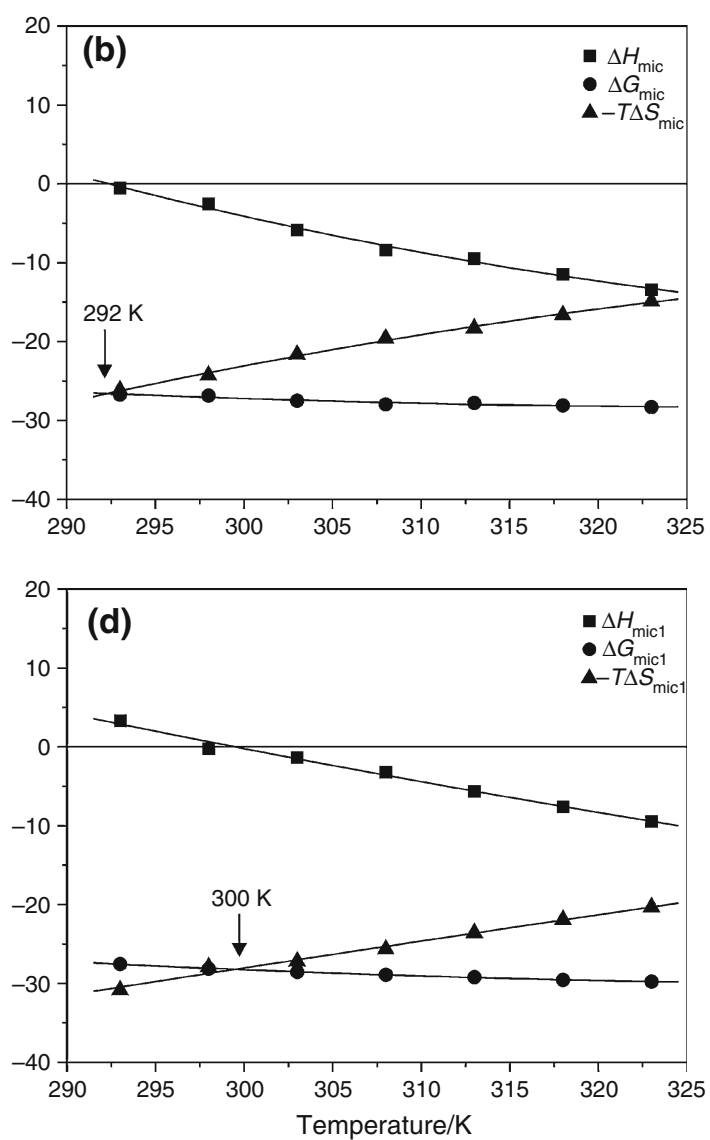

Fig. 4 Thermodynamic parameters for micellization of a DPC, b SDS, c 9:1 DPC/SDS, and d 5:1 DPC/SDS as a function of temperature. Intersection of the second polynomial approximation of $\Delta G_{\mathrm{mic}}$ and $-T \Delta S_{\mathrm{mic}}$ versus temperature gives the transition temperature, $T_{0}$ 


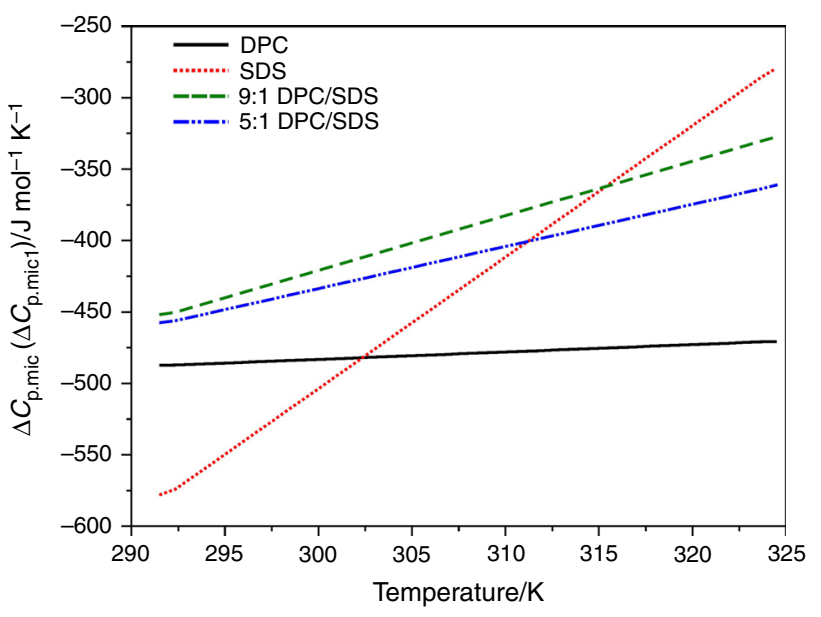

Fig. 5 Changes in heat capacity observed for the micellization of surfactants as a function of temperature. The lines were obtained from a second-order polynomial fit for the micellization enthalpies

$\Delta C_{\mathrm{p}, \text { mic }}$ values become less negative with increasing temperature as $\Delta H_{\text {mic }}$ is not a linear function of temperature. At $298 \mathrm{~K}, \Delta C_{\mathrm{p}, \mathrm{mic}}$ are $-484,-521,-428$, and $-439 \mathrm{~J} \mathrm{~mol}^{-1} \mathrm{~K}^{-1}$ for DPC, SDS, 9:1 DPC/SDS, and 5:1 DPC/SDS, respectively. This means that about $15,16,13$, and 13 hydrogen atoms are not in contact with water in DPC, SDS, 9:1 DPC/SDS, and 5:1 DPC/SDS, respectively, which corresponds to the terminal methyl group plus six for pure DPC and SDS micelles and five methylene groups for both mixed micelles. Thus, the DPC/SDS mixed micelles seem to be hydrated deeper by one methylene group than the pure ones, which may suggest either a less compact structure or a lower aggregation number. However, these calculations are oversimplified because they are based on the assumption that only the hydrophobic chain affects the heat capacity. Meanwhile, the heat capacity change during the micellization can also be related to changes in the so-called hydrophilic hydration. During the micellization process, the condensation of ions onto micelles reduces the number of water molecules in the solvation shell of both counterions and polar headgroups since the counterions can share hydration water with the headgroups [31]. "Hydrophilic dehydration" produces an increase in the heat capacity and behaves in an opposite manner to "hydrophobic dehydration", which decreases the heat capacity. For pure zwitterionic DPC, the changes in $\Delta C_{\mathrm{p} \text {,mic }}$ value are negligible within the whole temperature range, which suggests that $\Delta C_{\mathrm{p} \text {,mic }}$ can be assumed to solely reflect the change in the exposure of hydrophobic tails to water during the micellization. The largest changes in $\Delta C_{\mathrm{p} \text {,mic }}$ with increasing temperature are observed for pure anionic SDS, where the "hydrophilic dehydration" is associated with dehydration of sodium ions and sulfate polar headgroups. In the case of the DPC/SDS mixed micelles, besides the possible dehydration of sodium ions and sulfate headgroups, also the dehydration of phosphate group should be taken into account. The partitioning of the SDS into the zwitterionic DPC micelle may result in the formation of a salt bridge between the quaternary amine group of DPC and negatively charged sulfate group of SDS. Consequently, the sodium ions can bind to the phosphate group of DPC. In this case, dehydration of phosphate groups may contribute to $\Delta C_{\mathrm{p}, \text { mic }}$. The change in heat capacity for sodium ions during dehydration was estimated to be $42 \mathrm{~J} \mathrm{~K}^{-1} \mathrm{~mol}^{-1}$ [38]. Hence, assuming that the counterion binding degree of SDS is ca. 0.7 [32], the heat capacity associated with dehydration of sodium ions is only $6 \%$ of the total contribution of $\Delta C_{\mathrm{p} \text {,mic }}$ in the micellization process of SDS in PBS buffer. In turn, there is no quantitative data on the heat capacity associated with dehydration of sulfate and phosphate groups of SDS and DPC, respectively. In this case, we can compare only values calculated for dehydration of the sulfate $\left(\mathrm{SO}_{4}^{2-}\right)$ and phosphate $\left(\mathrm{PO}_{4}^{3-}\right)$ ions and these are, respectively, 193 and $341 \mathrm{~J} \mathrm{~K}^{-1} \mathrm{~mol}^{-1}$ [38]. Considering these data, an obvious impact of "hydrophilic dehydration" on the heat capacity change during micellization of the SDS and DPC/SDS mixed micelle is suggested. This would reduce the number of methylene groups in contact with water in SDS-containing micelles. Following this consideration, less negative $\Delta C_{\mathrm{p}, \text { mic }}$ values obtained for the DPC/SDS mixed micelle than for other micelles can be a consequence of the overcompensating effect of the enhanced "hydrophilic dehydration".

\section{Enthalpy-entropy compensation}

The free energy $\Delta G_{\text {mic }}$ is negative over the whole temperature range studied (Tab.1), which means that micellization is thermodynamically favorable and occurs spontaneously. At lower temperature, the contribution of the enthalpy to the Gibbs energy is small compared to the entropy term. However, as mentioned above, with increasing temperature, the enthalpy of micellization becomes more negative and its contribution to the Gibbs energy increases. In turn, the entropy of micellization is positive and decreases at elevated temperature. Thus, its contribution to the Gibbs energy also decreases. The $\Delta G_{\text {mic }}$ exhibits only slightly temperature dependence (Fig. 4) which results from the enthalpy-entropy compensation [39].

The enthalpy-entropy compensation plots for the micelles studied are shown in Fig. 6. This linear relationship can be ascribed by equation [40]:

$\Delta H_{\text {mic }}=T_{\mathrm{c}} \Delta S_{\text {mic }}+\Delta H_{\text {mic }}^{*}$ 


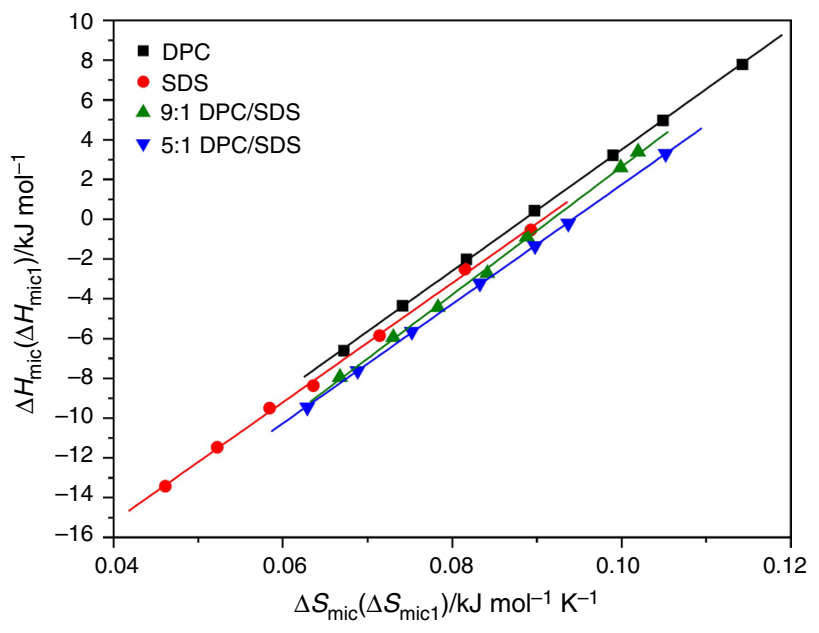

Fig. 6 Enthalpy-entropy compensation plot for the micelles studied

The slope in the equation is the compensation temperature $\left(T_{\mathrm{c}}\right)$ and represents solute-solvent interactions. It can be interpreted as a measure of dehydration of the hydrocarbon chain (desolvation part of micellization). The intercept, $\Delta H_{\mathrm{mic}}^{*}$, characterizes the solute-solute interactions, i.e., aggregation of hydrocarbon tails into micelle (chemical part of micellization) [40]. The $T_{\mathrm{c}}$ values are $305 \mathrm{~K}, 300 \mathrm{~K}, 322 \mathrm{~K}$, and $300 \mathrm{~K}$ for DPC, SDS, 9:1 DPC/SDS, and 5:1 DPC/SDS, respectively, and are consistent with those reported for various surfactants [39]. The higher $T_{\mathrm{c}}$ value found for the 9:1 DPC/SDS micelle than for the other systems can be attributed to some difference in the water-surfactant interaction mode during micellization process. The corresponding $\Delta H_{\mathrm{mic}}^{*}$ values are $-27.0,-27.2,-29.5$, and $-28.3 \mathrm{~kJ} \mathrm{~mol}^{-1}$. As seen, the difference between zwitterionic DPC and anionic SDS is here negligible, thus supporting the opinion that under the condition of a fixed number of carbon atoms in a straight hydrocarbon tail, the surfactants with different polar headgroups exhibit a small variation in the intercept $\Delta H_{\text {mic }}^{*}$ due to relatively small effects of the hydrophilic group on the chemical part of the micellization process [41]. However, the slight decrease in $\Delta H_{\text {mic }}^{*}$ upon addition of SDS to DPC can be explained in terms of a higher stability of the zwitanionic micelles. Given that $\Delta H_{\text {mic }}^{*}$ accounts for the solute-solute interactions, the observed decrease in $\Delta H_{\text {mic }}^{*}$ corresponds to enhancement of these interactions.

\section{Mixed micelles in unbuffered solution}

The ITC measurements of DPC-containing micelles were also taken in unbuffered solution at $298 \mathrm{~K}$. It is interesting to note that only a single-step micellization process is observed with the mixed micelles under these conditions (Fig. 7). The cmc values determined by titration calorimetry in the buffer-free solution are comparable with those obtained by the surface tension method (Table 2 and Fig. 1S, Supplementary Material). The results indicate that the $c m c$ of the zwitterionic DPC as well as zwitanionic 5:1 DPC/SDS decreases with an increasing ionic strength of solution. The presence of the electrolyte generally results in a decrease in the $c m c$ of most surfactants, wherein the strongest effect could be noticed with the ionic ones. An increase in the solution ionic strength results in an effective screening of the headgroup repulsion leading to tight packing and reduction in $\mathrm{cmc}$. As reported elsewhere, the cmc of pure SDS in the presence of electrolytes may decrease up to eight times as compared to that in water $[23,30-32]$. In the case of zwitterionic DPC, this effect is less pronounced because of the overall neutral charge of the surfactant. Therefore, it is proposed that the drop in the cmc of DPC might be due to salting out and the implication of phosphocholine headgroups in organization of the surrounding water molecules [42]. It is worth noting that for the 9:1 DPC/SDS system, the change in $c m c$ observed upon transfer from the unbuffered to buffered solution is negligible (taking into account $c m c 1$ in PBS).

\section{Surfactant-surfactant interactions in the mixed micelle}

Interaction parameter $\beta$ obtained from the Rubingh's regular solution theory (RST) [43] has commonly been used to characterize the strength and nature of interactions between two different surfactants in nonideal mixtures. Positive values of $\beta$ correspond to repulsive electrostatic interactions, preventing the micelle formation, whereas the negative values indicate attractive electrostatic interactions favorable for the micelle formation. For the nonideal mixing behavior, parameter $\beta$ can be calculated from the following expression [43]:

$\beta=\ln \left(\frac{\alpha_{\mathrm{SDS}} \cdot c m c_{\mathrm{mix}}}{\chi_{\mathrm{SDS}} \cdot c m c_{\mathrm{SDS}}}\right)\left(1-\chi_{\mathrm{SDS}}\right)^{-2}$

where $\alpha_{\text {SDS }}$ is the total mole fraction of SDS and $\chi_{\text {SDS }}$ is the mole fraction of SDS in the mixed micelle. $\chi_{\text {SDS }}$ parameter can be calculated by solving the following numerical equation [43]:

$$
\frac{\chi_{\mathrm{SDS}}^{2} \ln \left(\frac{\alpha_{\mathrm{SDS}} \cdot c m c_{\operatorname{mix}}}{\chi_{\mathrm{SDS}} \cdot c m c_{\mathrm{SDS}}}\right)}{\left(1-\chi_{\mathrm{SDS}}\right)^{2} \ln \left(\frac{\left(1-\alpha_{\mathrm{SDS}}\right) c m c_{\operatorname{mix}}}{\left(1-\chi_{\mathrm{SDS}}\right) c m c_{\mathrm{DPC}}}\right)}=1
$$

The knowledge of $\beta$ and $\chi_{\text {SDS }}$ parameters allows us to determine the activity coefficients $f_{\mathrm{SDS}}$ and $f_{\mathrm{DPC}}$ by [43]: 
Fig. 7 ITC experiments for the determination of the $\mathrm{cmc}$ of the DPC/SDS mixed micelles in aqueous buffer-free solution at 298 K. Top panels correspond to the experimental raw data (heat flow vs. time), while bottom ones correspond to the integrated injection heats and the first derivative of the ITC titration curve calculated from the interpolated values. In contrast to the buffer solution, a single-step aggregation mechanism is observed for the mixed micelles at $298 \mathrm{~K}$

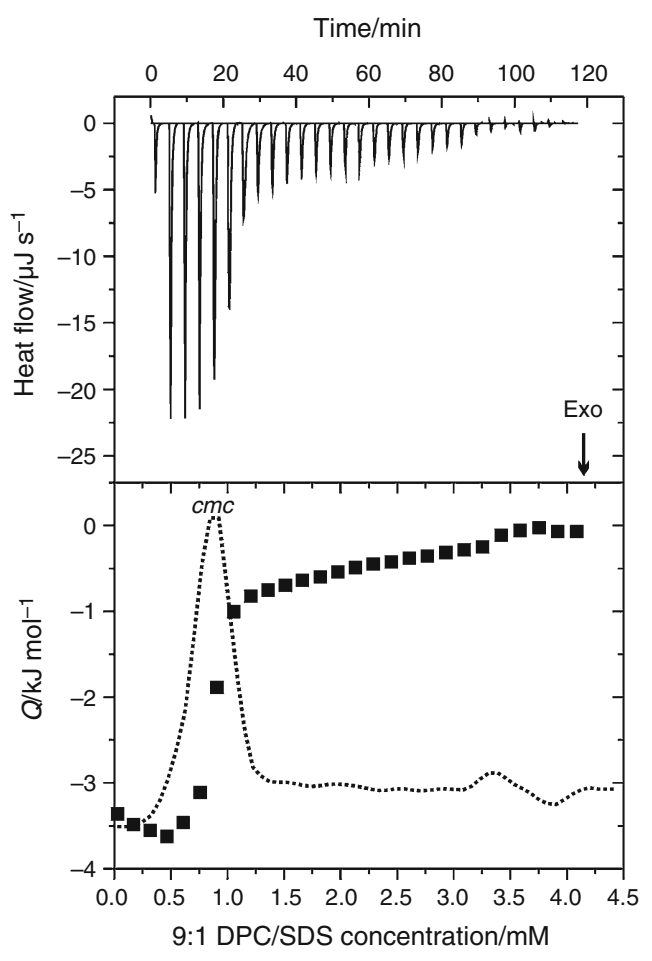

Table 2 A summary of critical micelle concentration values determined for DPC-containing micelles from surface tension method and ITC in water at $298 \mathrm{~K}$

\begin{tabular}{|c|c|c|c|c|c|c|c|c|}
\hline \multirow[t]{3}{*}{ Micelle } & \multirow{2}{*}{\multicolumn{2}{|c|}{$\frac{\text { Surface tension }}{\text { Water }}$}} & \multicolumn{4}{|l|}{ ITC } & \multicolumn{2}{|l|}{$\mathrm{NMR}^{\mathrm{b}}$} \\
\hline & & & \multicolumn{2}{|l|}{ Water } & \multicolumn{2}{|l|}{ PBS } & \multirow{2}{*}{$\begin{array}{l}\text { Water } \\
c m c / m M\end{array}$} & \multirow{2}{*}{$\begin{array}{l}\text { PBS } \\
c m c / m M\end{array}$} \\
\hline & $\mathrm{cmc} / \mathrm{mM}$ & $\sigma_{\mathrm{cmc}} / \mathrm{mN} \mathrm{m}^{-1}$ & $\mathrm{cmc} / \mathrm{mM}$ & $\Delta H_{\text {mic }} / \mathrm{kJ} \mathrm{mol}^{-1}$ & $\mathrm{cmc} / \mathrm{mM}$ & $\Delta H_{\text {mic }} / \mathrm{kJ} \mathrm{mol}^{-1}$ & & \\
\hline DPC & 1.33 & 41.12 & 1.44 & 5.23 & 1.36 & 4.97 & 1.52 & 1.36 \\
\hline 9:1 DPC/SDS & 0.94 & 39.36 & 0.89 & 2.80 & $0.94(1.60)$ & $2.58^{\mathrm{a}}$ & 1.32 & 1.35 \\
\hline 5:1 DPC/SDS & 0.84 & 38.48 & 0.85 & 2.22 & $0.65(1.18)$ & -0.19 (1.79) & - & - \\
\hline
\end{tabular}

To illustrate the changes induced by PBS, the $c m c$ and $\Delta H_{\text {mic }}$ values were repeated from Table 1 . The $c m c$ and $\Delta H_{\text {mic }}$ values in parentheses correspond to the second step of micellization process

$\sigma_{\mathrm{cmc}}$ denotes the surface tension of surfactant solution in $\mathrm{cmc}$ point

${ }^{\text {a }}$ Both steps of the micellization processes in the DPC/SDS mixed micelles are endothermic, and due to proximity of both $\mathrm{cmc}$ values, the separation of two reaction enthalpies is difficult

b Taken from [30]

$$
\begin{aligned}
& f_{\mathrm{SDS}}=\exp \left[\beta\left(1-\chi_{\mathrm{SDS}}\right)^{2}\right] \\
& f_{\mathrm{DPC}}=\exp \left[\beta \chi_{\mathrm{SDS}}^{2}\right]
\end{aligned}
$$

Accordingly, experimental values of $\mathrm{cmc}$ of two surfactants along with their activity coefficients in the mixture allow us to calculate $c m c_{\text {mix }}$ of a nonideal mixed micellar system [43]:

$\frac{1}{c m c_{\text {mix }}}=\frac{\alpha_{\mathrm{SDS}}}{f_{\mathrm{SDS}} \cdot c m c_{\mathrm{SDS}}}+\frac{\left(1-\alpha_{\mathrm{SDS}}\right)}{f_{\mathrm{DPC}} \cdot c m c_{\mathrm{DPC}}}$
For an ideally mixed system, activity coefficients $f_{\text {SDS }}$ and $f_{\text {DPC }}$ are equal to 1 and Eq. (9) is reduced to give a Clint's model [44]:

$\frac{1}{c m c_{\operatorname{mix}}^{\text {ideal }}}=\frac{\alpha_{\mathrm{SDS}}}{c m c_{\mathrm{SDS}}}+\frac{\left(1-\alpha_{\mathrm{SDS}}\right)}{c m c_{\mathrm{DPC}}}$

For the mixed systems studied, mole fractions of SDS in the mixed micelles $\left(\chi_{\mathrm{SDS}}\right)$, interaction parameter $(\beta)$, and activity coefficients $\left(f_{\mathrm{SDS}}\right.$ and $\left.f_{\mathrm{DPC}}\right)$ are listed in Table 1S (Supplementary Material). The mole fraction of 
$\operatorname{SDS}\left(\chi_{\mathrm{SDS}}\right)$ is always greater than the total mole fraction $\alpha$ of 0.1 and 0.17 for the 9:1 DPC/SDS and 5:1 DPC/SDS mixed micelles, respectively. This results from nonideal behavior and indicates that more SDS is transferred into the mixed micelle than is expected for an ideally mixed system. Over the whole temperature range studied, $\beta$ parameters are negative and their absolute values are greater than those of $\ln \left(\mathrm{cmc}_{\mathrm{SDS}} / \mathrm{cmc}_{\mathrm{DPC}}\right)$ for both mixed systems. This clearly indicates that SDS and DPC are synergetic in the formation of the mixed micelle in PBS solution. The synergy could also arise from the remarkable deviation between the experimental $c m c$ values and those calculated for the ideally mixed system described by the Clint's model (Fig. 2S, Supplementary Material).

\section{NMR characterization of mixed micelles}

\section{Self-diffusion measurements}

To get some insight into the micelle's size upon mixing of two surfactants, translational diffusion coefficients $\left(D_{\mathrm{tr}}\right)$ were determined with the DPFGDSTE (double pulsed field gradient double stimulated echo) experiment for DPCcontaining micellar systems. Due to the two-step aggregation mechanism observed for the DPC/SDS mixed micelles over the temperature range 293-313 K, we decided to conduct self-diffusion measurements at three temperatures within that range and to compare them with the results obtained for pure DPC, which reveals only a singlestep aggregation mechanism. The signal attenuation as a function of diffusion gradients, $g^{2} \gamma^{2} \delta^{2}(\Delta-\delta / 3)$, associated with the choline methyl groups is mono-exponential for each studied system over the whole range of temperature (Fig. 3S, Supplementary Material). This results from free bulk diffusion (unrestricted system) with the diffusion coefficient independent of the diffusion time [45, 46].

The determined self-diffusion coefficients of the studied micelles together with the hydrodynamic radius $\left(R_{\mathrm{h}}\right)$ are given in Table $2 \mathrm{~S}$ (Supplementary Material). The radius was estimated based on experimentally determined $D_{\text {tr }}$ according to Stokes-Einstein's equation. The calculated values for the radius of the DPC micelle as well as its two mixtures with SDS vary between 32 and $36 \AA$. Thus, addition of SDS only slightly increases the size of the micelle. The results obtained for DPC are in excellent agreement with those of the previous measurements, where the diffusion coefficient for the $228 \mathrm{mM}$ DPC in a $10 \mathrm{mM}$ phosphate buffer solution was found to be $9.17 \times 10^{-11} \mathrm{~m}^{2} \mathrm{~s}^{-1}$ at $310 \mathrm{~K}$ [11]. When corrected for temperature due to viscosity differences, this leads to an equivalent of $D_{\mathrm{tr}}=9.87 \times 10^{-11} \mathrm{~m}^{2} \mathrm{~s}^{-1}$ at $313 \mathrm{~K}$. This value is close to that obtained for DPC at $313 \mathrm{~K}$ $\left(D_{\mathrm{tr}}=10.69 \times 10^{-11} \mathrm{~m}^{2} \mathrm{~s}^{-1}\right)$ in this study.

\section{Molecular mobility of DPC-containing micelles}

The ${ }^{1} \mathrm{H}$ spectrum of DPC displays six well-resolved signals (Fig. 4S, Supplementary Material). The ${ }^{1} \mathrm{H}$ NMR signals from the $\mathrm{C}_{3}-\mathrm{C}_{11}$ fragment of the hydrocarbon chain $(1.25 \mathrm{ppm})$ are strongly overlapped; therefore, average relaxation times were calculated for this range of chemical shifts. Furthermore, the signals from $\mathrm{H}_{2}, \mathrm{H}_{3}-\mathrm{H}_{11}$, and $\mathrm{H}_{12}$ protons of DPC overlap those arising from SDS in the DPC/SDS mixed micelles. Nevertheless, both surfactants have the same length of hydrocarbon chain and, in our opinion, the protons in the corresponding positions of the hydrocarbon chain of DPC and SDS can be treated as identical.

As follows from our data, the ${ }^{1} \mathrm{H} T_{1}$ profiles are similar for all the micellar systems studied at three temperatures (Fig. 5S, Supplementary Material). The data exhibit a plateau with an average value of $0.6-0.8 \mathrm{~s}$ for the ${ }^{1} \mathrm{H}$ signals within the polar fragment of DPC. The short $T_{1} \mathrm{~s}$ within the polar segment of the micelles are a consequence of its restricted mobility. Besides, small differences in the $T_{1}$ values suggest either that DPC headgroup is a compact entity or that the motions take place in a synchronized manner. Remarkably higher values of $T_{1}$ observed for the hydrogens of terminal methyl group suggest enhanced dynamics and flexibility.

Examination of the data of spin-spin relaxation $\left(T_{2}\right)$ shows that methylene protons on the polar headgroup ( $\mathrm{H} \beta$ and $\mathrm{H} \alpha)$ and $\mathrm{H} 1$ on the hydrocarbon chain in the micellar systems studied reveal a heterogeneous dynamic behavior reflected in a two-component $T_{2}$ relaxation (Figs. $6 \mathrm{~S}$ and 7S, Supplementary Material). An increase in the temperature results in a bi-exponential $T_{2}$ model for $\mathrm{H} 2$ methylene protons and the terminal methyl group. The choline methyl groups ( $3.23 \mathrm{ppm})$ and the methylene groups in the C3-C11 fragment of the hydrocarbon chain (the overlapped signal at $1.25 \mathrm{ppm}$ ) relax mono-exponentially within the temperature range studied (Fig. 7S, Supplementary Material). The biexponential ${ }^{1} \mathrm{H} T_{2}$ relaxations are characterized by a short component with $T_{2}$ of $15-80 \mathrm{~ms}$ and a long one with $T_{2}$ of 200-900 ms. A weighting factor for the fast-relaxing component ranges from 10 to $20 \%$ at $293 \mathrm{~K}$ and increases approximately three times at $313 \mathrm{~K}$. The fractions of the fastrelaxing methylene protons on the polar headgroup $(\mathrm{H} \beta$ and $\mathrm{H} \alpha$ ) and $\mathrm{H} 1$ on the hydrocarbon chain reveal similar weighting factors of about 15,25 , and $50 \%$ at 293,303 , and $313 \mathrm{~K}$, respectively. A significant difference is observed for $\mathrm{H} 2$ at $313 \mathrm{~K}$ (at 293 and $303 \mathrm{~K} \mathrm{H} 2$ protons relax monoexponentially), where the weighting factor of the fast-relaxing component is reduced by ca. $20-30 \%$ as compared to 
those of $\mathrm{H} \beta, \mathrm{H} \alpha$, and $\mathrm{H} 1$. The $T_{2}$ decay curve of the terminal methyl group exhibits bi-exponentiality only at $313 \mathrm{~K}$, similar to $\mathrm{H} 2$ methylene protons, with the weighting factor of the fast-relaxing component in the range of $10-15 \%$. Since the $T_{2}$ values could previously be treated as a measure of spatial distribution of identical protons [47], one could construct a picture of chain packing in the micelle based on the ${ }^{1} \mathrm{H} T_{2}$ values of a particular part of surfactant molecule. Moreover, knowing that ${ }^{1} \mathrm{H}$ spin-spin relaxation is sensitive to molecular motion, bi-exponentiality of $T_{2}$ relaxation time indicates existence of two types of molecular motions in solution. The relaxation component with a short $T_{2}$ value can be attributed to the restricted chain segments, i.e., undergoing a slow motion, whereas that with the long $T_{2}$ value corresponds to the segments that have a fast motion due to higher mobility, e.g., the more hydrated segments [47-49]. Following this interpretation, we concluded that the monoexponential behavior of the choline methyl groups at each temperature studied with a high $T_{2}$ value of 415-670 ms, irrespective of the micelle composition, indicates protons exposed to aqueous phase. The decrease in the $T_{2}$ values for subsequent protons indicates a tighter packing as expected for groups either approaching or forming hydrophobic core of the micelle. Thus, the $T_{2 \mathrm{~s}}$ values observed for the $\mathrm{H} 1$ and $\mathrm{H} 2$ protons are by more than twice lower than those of the $\left(\mathrm{C}_{\gamma} \mathrm{H}_{3}\right)_{3}$ methyl groups. Some inconsistencies appear with the $\mathrm{H} \beta$ and $\mathrm{H} \alpha$ methylene protons at $313 \mathrm{~K}$, where the slowrelaxing component is characterized by $T_{2 \mathrm{~s}}$ values higher than those calculated for the choline methyl groups (Fig. 7S, Supplementary Material). However, as seen, both $T_{2}$ values are subjected to considerable error. The $T_{2}$ value of the terminal methyl group in the hydrocarbon chain is distinctly greater than those for the remaining segments of the hydrophobic micellar core. This indicates its greater mobility. This conclusion is compatible with theoretical studies on DPC micelle [50]. The theoretical DPC model indicates that the surfactant chains are significantly disordered and that the terminal methyl groups of the surfactant alkyl chains are broadly distributed throughout the micelle's volume. Besides, the tendency of the hydrocarbon chain to "swing" toward the interfacial region increases the hydration number of the terminal methyl group. In turn, the change in the relaxation behavior of protons with increasing temperature, i.e., increase in the weighting factors for the fastrelaxing components, indicates gradual dehydration of the micelle making it more compact.

The $T_{1} / T_{2}$ ratio can be related to the degree of restricted motion of respective nuclei. Thus, the variation in the $T_{1} / T_{2}$ ratios along the whole DPC molecule can correlate with the molecular packing of a particular part of the surfactant in the micelle. The $T_{1} / T_{2}$ ratios were calculated by using $T_{2 \mathrm{~s}}$ values for the bi-exponentially relaxing protons. We considered the data obtained at $293 \mathrm{~K}$ because at this temperature, the slow-relaxing component of the bi-exponentially decaying protons predominates with weighting factors greater than $80 \%$.

${ }^{1} \mathrm{H} T_{1} / T_{2}$ ratios are remarkably larger for the hydrocarbon chains than for polar headgroups of DPC (Fig. 8), indicating more restricted motions of the former and their tighter packing. The largest $T_{1} / T_{2}$ ratio was determined for the $\mathrm{H} 1$ methylene protons, and it gradually decreased upon going to the end of the hydrocarbon chain. This suggests that $\mathrm{H} 1$ protons are the main constituent of the rigid interfacial layer core protecting the penetration of water into the hydrophobic interior. An addition of SDS leads to an increase in the $T_{1} / T_{2}$ ratios beginning from $\mathrm{H} \alpha$ methylene protons in the polar headgroup of DPC and ending at the terminal methyl group of the hydrocarbon chain, while the $T_{1} / T_{2}$ ratios of the choline methyl groups and $\mathrm{H} \beta$ methylene protons of the phosphocholine headgroup remain unchanged. These data suggest that the hydrocarbon chain of SDS is more or less covered with that of DPC, as expected based on their identical lengths.

Mixing SDS with DPC shortens only slightly the $T_{1}$ values of the ${ }^{31} \mathrm{P}$ nucleus. The change variations do not exceed $6 \%$ (Table 3). A much greater reduction is seen in the transverse relaxation time $T_{2}$, where a decrease in the range of $9-44 \%$ has been noticed. The reduction in $T_{2}$ values of ${ }^{31} \mathrm{P}$ increases upon raising the SDS concentration. Since $T_{2}$ is sensitive to variations of both overall and local correlation times, whereas $T_{1}$ depends mainly on local correlation times, the results indicate that the average local dynamics of the DPC molecule is not affected by the presence of SDS. However, its presence leads to an increase in the overall correlation time, i.e., in the hydrodynamic volume of the micelle.

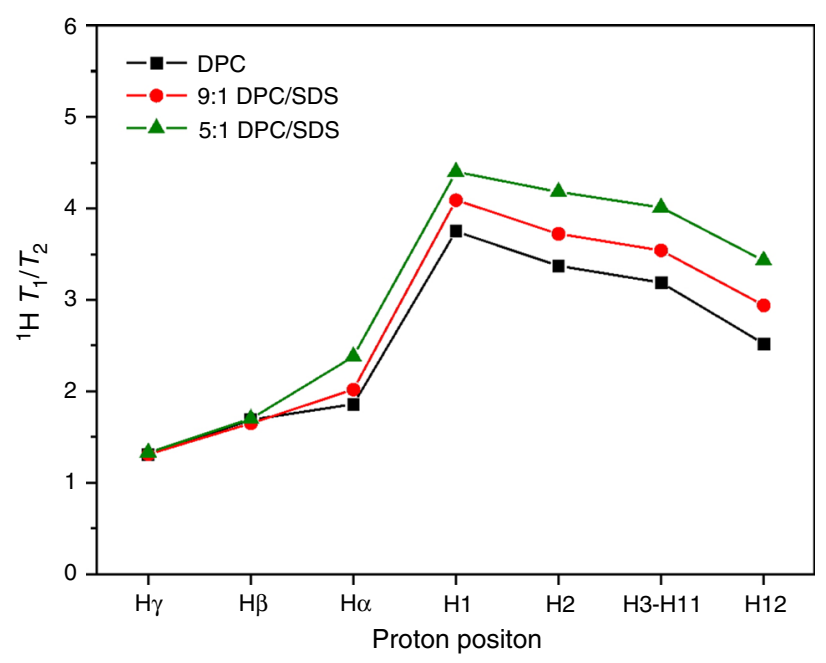

Fig. $8{ }^{1} \mathrm{H} T_{1} / T_{2}$ ratios in pure DPC, 9:1 DPC/SDS, and 5:1 DPC/SDS micelles at $293 \mathrm{~K}$ 
Table 3 Longitudinal $\left(T_{1}\right)$ and transverse $\left(T_{2}\right)$ relaxation times of the phosphorus ${ }^{31} \mathrm{P}$ in the DPC and DPC/SDS mixed micelles at different mole ratios with a total concentration of $150 \mathrm{mM}$

\begin{tabular}{llll}
\hline Micelle & $T_{1} / \mathrm{s}$ & $T_{2} / \mathrm{s}$ & $T_{1} / T_{2}$ \\
\hline$T=293 \mathrm{~K}$ & & & \\
DPC & 0.98 & 0.22 & 4.47 \\
9:1 DPC/SDS & 0.99 & 0.20 & 4.97 \\
5:1 DPC/SDS & 0.96 & 0.17 & 5.70 \\
$T=303 \mathrm{~K}$ & & & \\
DPC & 1.16 & 0.24 & 4.97 \\
9:1 DPC/SDS & 1.10 & 0.20 & 5.38 \\
5:1 DPC/SDS & 1.09 & 0.16 & 7.23 \\
$T=313 \mathrm{~K}$ & & & \\
DPC & 1.37 & 0.23 & 5.70 \\
9:1 DPC/SDS & 1.31 & 0.18 & 6.91 \\
5:1 DPC/SDS & 1.30 & 0.13 & 9.65 \\
\hline
\end{tabular}

The standard deviation did not exceed $5 \%$

\section{Conclusions}

In the present paper, we studied the micellization of DPC, SDS, and the DPC/SDS mixed micelles at two molar ratios 9:1 and 5:1 using isothermal calorimetric titration (ITC). NMR relaxation measurements were used to explore the molecular mobility of the DPC-containing micelles. The diffusion measurements were taken to determine the size of the micelles at a high concentration, well above their $\mathrm{cmc}$.

According to ITC, the DPC/SDS mixed systems show the tendency to form two kinds of micelles in PBS solution at lower temperatures. At $293 \mathrm{~K}$, both steps of micellization are endothermic. An increase in the temperature causes an endothermic-to-exothermic transition only of the first step of micellization, while the second step becomes still endothermic. Further increase in temperature results in only a single micellization process with exothermic heat change. This reveals the formation of only one kind of the micelle at a higher temperature. Existence of two structures is usually attributed to either changes in the composition in the mixed micelles or transition of the shape with the increase in a total surfactant concentration $[20,28,29]$. As the size of the pure DPC and the DPC/SDS mixed micelles is comparable, as determined by diffusion measurements, we suppose that both kinds of the mixed micelles found at lower temperatures by ITC differ from each other in the content of individual surfactants. In our opinion, the $c m c 1$ corresponds to the mixed micelle with a higher content of SDS. It is worth noting that in unbuffered aqueous solution, only one kind of the micelle occurs for the mixture of DPC and SDS and this phenomenon requires further investigations.
Acknowledgements This work was supported in part by the University of Gdańsk (DS 530-8455-D500-14) and partially supported by Polish National Centre for Research and Development under research Grant Number 178479 (Contract Number PBS1/A9/ 13/2012) (for KS and IZ). The preliminary NMR measurements for micelles were taken in the Slovenian NMR Centre within the PF7 EAST-NMR project (Contract Number 228461).

Open Access This article is distributed under the terms of the Creative Commons Attribution 4.0 International License (http://creative commons.org/licenses/by/4.0/), which permits unrestricted use, distribution, and reproduction in any medium, provided you give appropriate credit to the original author(s) and the source, provide a link to the Creative Commons license, and indicate if changes were made.

\section{References}

1. Galdiero S, Falanga A, Cantisani M, Vitiello M, Morelli G, Galdiero M. Peptide-lipid interactions: experiments and applications. Int J Mol Sci. 2013;14:18758-89.

2. Langelaan DN, Rainey JK. Membrane catalysis of peptide-receptor binding. Biochem Cell Biol. 2010;88:203-10.

3. Sargent D, Schwyzer R. Membrane lipid phase as catalyst for peptide-receptor interactions. Proc Natl Acad Sci USA. 1986;83:5774-8.

4. Trabulo S, Cardoso AL, Mano M, De Lima MCP. Cell-penetrating peptides-mechanisms of cellular uptake and generation of delivery systems. Pharmaceuticals. 2010;3:961-93.

5. Yeaman MR, Yount NY. Mechanisms of antimicrobial peptide action and resistance. Pharmacol Rev. 2003;55:27-55.

6. Bader R, Lerch M, Zerbe O. NMR of membrane-associated peptides and proteins. In: Zerbe O, editor. BioNMR in Drug Research. Weinheim: Wiley-VCH; 2003. p. 95-117.

7. Warschawski DE, Arnold AA, Beaugrand M, Gravel A, Chartrand É, Marcotte I. Choosing membrane mimetics for NMR structural studies of transmembrane proteins. BBA-Biomembranes. 2011;1808:1957-74.

8. Damberg P, Jarvet J, Gräslund A. Micellar systems as solvents in peptide and protein structure determination. Methods Enzymol. 2001;339:271-85.

9. Marassi FM, Opella SJ. NMR structural studies of membrane proteins. Curr Opin Struct Biol. 1998;8:640-8.

10. Hsu S-TD, Breukink E, Bierbaum G, Sahl H-G, de Kruijff B, Kaptein R, van Nuland NA, Bonvin AM. NMR study of mersacidin and lipid II interaction in dodecylphosphocholine micelles. Conformational changes are a key to antimicrobial activity. J Biol Chem. 2003;278:13110-7.

11. Kallick DA, Tessmer MR, Watts CR, Li C-Y. The use of dodecylphosphocholine micelles in solution NMR. J Magn Reson Ser B. 1995;109:60-5.

12. Beswick V, Guerois R, Cordier-Ochsenbein F, Coïc Y-M, Huynh-Dinh T, Tostain J, Noël J-P, Sanson A, Neumann J-M. Dodecylphosphocholine micelles as a membrane-like environment: new results from NMR relaxation and paramagnetic relaxation enhancement analysis. Eur Biophys J. 1998;28:48-58.

13. Wang $X$, Wang $R$, Zheng Y, Sun L, Yu L, Jiao J. Interaction between zwitterionic surface activity ionic liquid and anionic surfactant: $\mathrm{Na}(+)$-driven wormlike micelles. J Phys Chem B. 2013;117:1886-95.

14. Hicks RP, Mones E, Kim H, Koser BW, Nichols DA, Bhattacharjee AK. Comparison of the conformation and electrostatic surface properties of magainin peptides bound to sodium dodecyl sulfate and dodecylphosphocholine micelles. Biopolymers. 2003;68:459-70. 
15. Langham AA, Waring AJ, Kaznessis YN. Comparison of interactions between beta-hairpin decapeptides and SDS/DPC micelles from experimental and simulation data. BMC Biochem. 2007;8:11.

16. Jaroniec CP, Kaufman JD, Stahl SJ, Viard M, Blumenthal R, Wingfield PT, Bax A. Structure and dynamics of micelle-associated human immunodeficiency virus gp41 fusion domain. Biochemistry. 2005;44:16167-80.

17. Weller K, Lauber S, Lerch M, Renaud A, Merkle HP, Zerbe O. Biophysical and biological studies of end-group-modified derivatives of Pep-1. Biochemistry. 2005;44:15799-811.

18. Scrima M, Campiglia P, Esposito C, Gomez-Monterrey I, Novellino E, D'Ursi AM. Obestatin conformational features: a strategy to unveil obestatin's biological role? Biochem Biophys Res Commun. 2007;363:500-5.

19. Stejskal E, Tanner J. Spin diffusion measurements: spin echoes in the presence of a time-dependent field gradient. J Chem Phys. 1965;42:288-92.

20. Hildebrand A, Garidel P, Neubert R, Blume A. Thermodynamics of demicellization of mixed micelles composed of sodium oleate and bile salts. Langmuir. 2004;20:320-8.

21. Feitosa E, Brazolin MRS, Zumstein Georgetto Naal RM, de Morais Del MPF, Lopes JR, Loh W, Vasilescu M. Structural organization of cetyltrimethylammonium sulfate in aqueous solution: The effect of $\mathrm{Na}_{2} \mathrm{SO}_{4}$. J Colloid Interface Sci. 2006;299:883-9.

22. Zana R. Critical micellization concentration of surfactants in aqueous solution and free energy of micellization. Langmuir. 1996;12:1208-11.

23. Paula S, Sues W, Tuchtenhagen J, Blume A. Thermodynamics of micelle formation as a function of temperature: a high sensitivity titration calorimetry study. J Phys Chem. 1995;99:11742-51.

24. Marsh D. Thermodynamics of phospholipid self-assembly. Biophys J. 2012;102:1079-87.

25. Prasad M, Moulik S, Palepu R. Self-aggregation of binary mixtures of alkyltriphenylphosphonium bromides: a critical assessment in favor of more than one kind of micelle formation. J Colloid Interface Sci. 2005;284:658-66.

26. Zimmels Y, Lin I. Stepwise association properties of some surfactant aqueous solutions. Colloid Polym Sci. 1974;252:594-612.

27. Majhi PR, Moulik SP. Energetics of micellization: reassessment by a high-sensitivity titration microcalorimeter. Langmuir. 1998;14:3986-90.

28. Du C, Cai D, Qin M, Zheng P, Hao Z, Yin T, Zhao J, Shen W. Thermodynamics of mixed surfactant solutions of $\mathrm{N}, \mathrm{N}^{\prime}$-bis (dimethyldodecyl)-1, 2-ethanediammoniumdibromide with 1-dodecyl-3-methylimidazolium bromide. J Phys Chem B. 2014;118: 1168-79.

29. Prasad M, Moulik S, MacDonald A, Palepu R. Self-aggregation of alkyl (C10-, C12-, C14-, and C16-) triphenyl phosphonium bromides and their 1: 1 molar mixtures in aqueous medium: a thermodynamic study. J Phys Chem B. 2004;108:355-62.

30. Manzo G, Carboni M, Rinaldi AC, Casu M, Scorciapino MA. Characterization of sodium dodecylsulphate and dodecylphosphocholine mixed micelles through NMR and dynamic light scattering. Magn Reson Chem. 2013;51:176-83.

31. Ropers M, Czichocki G, Brezesinski G. Counterion effect on the thermodynamics of micellization of alkyl sulfates. J Phys Chem B. $2003 ; 107: 5281-8$.
32. Taheri-Kafrani A, Bordbar A-K. Energitics of micellization of sodium n-dodecyl sulfate at physiological conditions using isothermal titration calorimetry. J Therm Anal Calorim. 2009;98: 567-75.

33. Makowska J, Wyrzykowski D, Pilarski B, Chmurzyński L. Thermodynamics of sodium dodecyl sulphate (SDS) micellization in the presence of some biologically relevant $\mathrm{pH}$ buffers. J Therm Anal Calorim. 2015;121:257-61.

34. Iwasaki T, Ogawa M, Esumi K, Meguro K. Interactions between betaine-type zwitterionic and anionic surfactants in mixed micelles. Langmuir. 1991;7:30-5.

35. Ghosh S, Khatua D, Dey J. Interaction between zwitterionic and anionic surfactants: spontaneous formation of zwitanionic vesicles. Langmuir. 2011;27:5184-92.

36. Li F, Li G-Z, Chen J-B. Synergism in mixed zwitterionic-anionic surfactant solutions and the aggregation numbers of the mixed micelles. Colloids Surf A. 1998;145:167-74.

37. Gill S, Wadsö I. An equation of state describing hydrophobic interactions. Proc Natl Acad Sci USA. 1976;73:2955-8.

38. Marcus Y. A simple empirical model describing the thermodynamics of hydration of ions of widely varying charges, sizes, and shapes. Biophys Chem. 1994;51:111-27.

39. Sugihara G, Hisatomi M. Enthalpy-entropy compensation phenomenon observed for different surfactants in aqueous solution. J Colloid Interface Sci. 1999;219:31-6.

40. Kumar S, Parikh K. Influence of temperature and salt on association and thermodynamic parameters of micellization of a cationic gemini surfactant. J Appl Sol Chem Model. 2012;1: $65-73$.

41. Chen L-J, Lin S-Y, Huang C-C. Effect of hydrophobic chain length of surfactants on enthalpy-entropy compensation of micellization. J Phys Chem. 1998;102:4350-6.

42. Palladino P, Rossi F, Ragone R. Effective critical micellar concentration of a zwitterionic detergent: a fluorimetric study on n-dodecyl phosphocholine. J Fluoresc. 2010;20:191-6.

43. Rubingh DN. Mixed micelle solutions. In: Mittal KL, editor. Solution chemistry of surfactants. New York: Plenum Press; 1979. p. 337-54.

44. Clint JH. Micellization of mixed nonionic surface active agents. J Chem Soc Faraday Trans. 1975;1(71):1327-34.

45. Barhoum S, Castillo R, Yethiraj A. Characterization of dynamics and internal structure of a mixed-surfactant wormlike micellar system using NMR and rheometry. Soft Matter. 2012;8:6950-7.

46. Szutkowski K, Klinowski J, Jurga S. NMR studies of restricted diffusion in lyotropic systems. Solid State Nucl Magn Reson. 2002;22:394-408.

47. Zhao S, Yuan H-Z, Yu J-Y, Du Y-R. Hydrocarbon chain packing in the micellar core of surfactants studied by $1 \mathrm{H}$ NMR relaxation. Colloid Polym Sci. 1998;276:1125-30.

48. He J, Tremblay L, Lacelle S, Zhao Y. Preparation of polymer single chain nanoparticles using intramolecular photodimerization of coumarin. Soft Matter. 2011;7:2380-6.

49. Ru G, Wang N, Huang S, Feng J. 1H HRMAS NMR study on phase transition of poly (N-isopropylacrylamide) gels with and without grafted comb-type chains. Macromolecules. 2009;42: 2074-8.

50. Wymore T, Gao X, Wong T. Molecular dynamics simulation of the structure and dynamics of a dodecylphosphocholine micelle in aqueous solution. J Mol Struct. 1999;485:195-210. 